\title{
Prognostic factors and failure patterns in non-metastatic nasopharyngeal carcinoma after intensity-modulated radiotherapy
}

Yan-Ping Mao ${ }^{1,2+}$, Ling-Long Tang ${ }^{1,2+}$, Lei Chen ${ }^{1,2}$, Ying Sun ${ }^{1,2}$, Zhen-Yu Qi ${ }^{1,2}$, Guan-Qun Zhou ${ }^{1,2}$, Li-Zhi Liu ${ }^{1,3}$, Li Li $i^{1,3}$, Ai-Hua Lin ${ }^{4}$ and Jun Ma ${ }^{1,2^{*}}$ (D)

\begin{abstract}
Background: The prognostic values of staging parameters require continual re-assessment amid changes in diagnostic and therapeutic methods. This study aimed to identify the prognostic factors and failure patterns of non-metastatic nasopharyngeal carcinoma (NPC) in the intensity-modulated radiotherapy (IMRT) era.

Methods: We reviewed the data from 749 patients with newly diagnosed, biopsy-proven, non-metastatic NPC in our cancer center (South China, an NPC endemic area) between January 2003 and December 2007. All patients underwent magnetic resonance imaging (MRI) before receiving IMRT. The actuarial survival rates were estimated using the Kaplan-Meier method, and survival curves were compared using the log-rank test. Multivariate analyses with the Cox proportional hazards model were used to test for the independent prognostic factors by backward eliminating insignificant explanatory variables.

Results: The 5-year occurrence rates of local failure, regional failure, locoregional failure, and distant failure were 5.4, 3.0, 7.4, and 17.4\%, respectively. The 5-year survival rates were as follows: local relapse-free survival, 94.6\%; nodal relapse-free survival, 97.0\%; distant metastasis-free survival, 82.6\%; disease-free survival, 75.1\%; and overall survival, $82.0 \%$. Multivariate Cox regression analysis revealed that orbit involvement was the only significant prognostic factor for local failure $(P=0.011)$. Parapharyngeal tumor extension, retropharyngeal lymph node involvement, and the laterality, longest diameter, and Ho's location of the cervical lymph nodes were significant prognostic factors for both distant failure and disease failure (all $P<0.05$ ). Intracranial extension had significant prognostic value for distant failure $(P=0.040)$.

Conclusions: The key failure pattern for NPC was distant metastasis in the IMRT era. With changes in diagnostic and therapeutic technologies as well as treatment modalities, the significant prognostic parameters for local control have also been altered substantially.
\end{abstract}

Keywords: Nasopharyngeal carcinoma, Intensity-modulated radiotherapy, Prognosis, Failure pattern, Tumor staging

\section{Background}

Nasopharyngeal carcinoma (NPC) is a challenge in oncology. Due to the deep-seated anatomic location and proximity of NPCs to critical structures, radical surgical

\footnotetext{
*Correspondence: majun@sysucc.org.cn

†Yan-Ping Mao and Ling-Long Tang contributed equally to this work

${ }^{1}$ State Key Laboratory of Oncology in South China, Collaborative Innovation Center for Cancer Medicine, Sun Yat-sen University Cancer Center, Guangzhou 510060, Guangdong, P. R. China

Full list of author information is available at the end of the article
}

resection is extremely difficult [1]. The introduction of radiotherapy has made this otherwise lethal malignant disease curable [1]. However, radiotherapy has undergone several different periods of development, and it was not until the advent of mega-voltage machines that a 5-year overall survival (OS) rate of $25 \%$ was first achieved, which marked the first major breakthrough in the treatment of NPC and established radiotherapy as the primary modality of choice for NPC [2]. 
Since that time, progressive improvements in the treatment outcomes of patients with NPC have been achieved. Initially, in the 1970-1980s, conventional radiotherapy alone resulted in similar outcomes in both the endemic and non-endemic areas, with 5-year OS rates of $48 \%-52 \%$, a cumulative local failure rate of $20 \%$, a cumulative regional failure rate of $14 \%$, and a cumulative distant metastasis rate of $19 \%$ [3, 4]. During the 1990s, rapid technological advances in imaging methods, computerized planning systems, and radiotherapy facilities and the accumulation of radiobiological knowledge that enabled schedule, dose, and fractionation optimization led to better outcomes after radiotherapy, with 5-year OS rates of $65 \%-74 \%$, a cumulative local failure rate of $12 \%$, a regional failure rate of $5 \%$, and a distant metastasis rate of $16 \%[5,6]$. However, local relapse and distant metastasis remained the two major causes of failure. Intensitymodulated radiotherapy (IMRT) was introduced in the 2000 s and represented a major breakthrough in the radiotherapeutic management of NPC. Due to the dosimetric advantages of IMRT combined with the use of magnetic resonance imaging (MRI)-guided tumor volume delineation and concurrent chemotherapy, local control rate in NPC patients has improved significantly $[7,8]$.

With these changes in diagnostic and therapeutic technologies, the significant prognostic parameters that have been identified in patients treated with conventional radiotherapy may also have been altered substantially. Therefore, caution must be applied when applying prognostic factors to predict prognosis and guide treatment strategies in patients treated using these modern, more effective treatment modalities [9]. Thus, the prognostic values of staging parameters require continual re-assessment amid these changes in diagnostic and therapeutic methods. However, few studies have systematically investigated the prognostic factors in NPC in the IMRT era. We performed a retrospective systematic evaluation of a large cohort of patients with NPC who were treated with IMRT at the Sun Yat-sen University Cancer Center in South China, an NPC endemic area. The aim of this study was to identify the key failure patterns and prognostic factors to improve the treatment of patients with NPC and provide a baseline for future studies.

\section{Methods}

\section{Patient cohort}

We reviewed the medical records of all patients with NPC treated with IMRT at the Sun Yat-sen University Cancer Center between January 2003 and December 2007. We included all patients with newly diagnosed, non-metastatic, histologically confirmed disease. All patients were staged using a standard protocol [10] comprising a complete disease history, physical examination, hematology and biochemistry profiles, fiberoptic nasopharyngoscopy, MRI of the neck and nasopharynx, chest radiography, abdominal sonography, and a whole-body bone scan using single-photon emission computed tomography (SPECT). All MRI materials and clinical records were reviewed to minimize heterogeneity in restaging. The study was reviewed and approved by the ethics committee of the Sun Yat-sen University Cancer Center.

\section{Image assessment and criteria for staging parameters}

Two radiologists specializing in head and neck cancers evaluated all scans separately. All parameters included in the TNM staging were re-assessed, and any disagreements were resolved by consensus. Parapharyngeal tumor extension was identified as a direct tumor extension through the pharyngobasilar fascia [11]. Nasal cavity extension was defined as tumor extension beyond the bony nasal septum [12]. Oropharyngeal extension was defined as the primary tumor extension of the mucosa or submucosal tissue below the plane of the superior surface of the soft palate or the C1-C2 interspace [13]. Hypopharyngeal extension was defined as the primary tumor extension of the mucosa or submucosal tissue to the superior border of the epiglottis or lower margin of the C3 interspace [14]. The skull base included the clivus, pterygoid bones, body of the sphenoid, apices of the petrous temporal bones, sphenoid wings, upper cervical spine, and skull base foramina and fissures [15]. The criterion for orbit extension was tumor extension into the orbital apex, inferior orbital fissure, or superior orbital fissure [16]. Involvement of the cavernous sinus, brain tissue, cistern, or dural meninges was interpreted as intracranial extension [17].

The diagnostic criteria for metastatic lymphadenopathy [18] include the following: (a) lateral retropharyngeal lymph nodes with a minimal axial diameter in the largest plane of an individual node of at least $5 \mathrm{~mm}$ and any node observed in the median retropharyngeal group, lymph nodes with a minimal axial diameter of at least $11 \mathrm{~mm}$ in the jugulodigastric region and $10 \mathrm{~mm}$ for all other cervical nodes; (b) lymph nodes of any size with central necrosis or a contrast-enhanced rim; (c) nodal groups, defined as the presence of three or more contiguous and confluent lymph nodes, each of which with a minimal axial diameter of 8-10 $\mathrm{mm}$; and/or (d) lymph nodes of any size with extracapsular spread, the presence of indistinct nodal margins, irregular nodal capsular enhancement, or infiltration into the adjacent fat or muscle.

\section{Treatment}

All patients underwent radical radiotherapy. The nasopharyngeal and upper neck tumor volumes were treated using IMRT for the entire treatment course. The lower 
neck was treated with a conventional anterior or anteroposterior opposing cervical technique. The prescribed doses were $68 \mathrm{~Gy}$ to the planning target volume of the primary tumor (PTV-P), 60-68 Gy to the PTV of the nodes (PTV-N), 60 Gy to the high-risk regions (PTV1), and 54 Gy to the low-risk regions (PTV2) in 30 fractions over 6 weeks. The anterior split-neck field received 50-60 Gy irradiation in 2-Gy fractions. The irradiation was delivered once daily for 5 days per week. Further details of the IMRT techniques used at our cancer center have been previously reported [10].

During the study period, concurrent chemoradiotherapy was recommended for stage III to IVa-b disease, and no chemotherapy was recommended for stage I to IIa disease (as defined using the 6th edition of the International Union against Cancer/American Joint Committee on Cancer [UICC/AJCC] staging system). However, some patients with stage III to IVa-b disease also received neoadjuvant or adjuvant chemotherapy, and some patients with stage IIb disease received concurrent chemoradiotherapy. Neoadjuvant or adjuvant chemotherapy consisted of cisplatin $\left(80-100 \mathrm{mg} / \mathrm{m}^{2}\right)$ with either 5 -fluorouracil $\left(800-1000 \mathrm{mg} / \mathrm{m}^{2}\right)$ or taxanes (paclitaxel [135$\left.175 \mathrm{mg} / \mathrm{m}^{2}\right]$ or docetaxel [70-75 $\left.\mathrm{mg} / \mathrm{m}^{2}\right]$ ) every 3 weeks for 3 cycles. Concomitant chemotherapy consisted of cisplatin $\left(80-100 \mathrm{mg} / \mathrm{m}^{2}\right)$ given on weeks 1 , 4 , and 7 of radiotherapy or cisplatin $\left(30-40 \mathrm{mg} / \mathrm{m}^{2}\right)$ given weekly. When possible, salvage treatments, such as intracavitary brachytherapy, surgery, and chemotherapy, were provided in the event of documented relapse or persistent disease.

\section{Follow-up}

Treatment responses and toxicity were assessed in each patient every week during treatment, every $2-3$ months during the first 2 years after treatment, and then every 3-6 months during the following 3 years. Endoscopy, computed tomography (CT), or MRI scans of the head and neck were also performed every 3 months in the first year and annually thereafter. Additional tests were performed when indicated to evaluate local or distant failure. The last follow-up time was 30th August 2013.

\section{Statistical analysis}

All analyses were performed using SPSS version 16.0 (SPSS, Chicago, IL, USA). The actuarial rates were estimated using the Kaplan-Meier method, and the survival curves were compared using the log-rank test. The following endpoints (measured from the start of treatment to the first defined event) were estimated: local relapsefree survival (LRFS), nodal relapse-free survival (NRFS), distant metastasis-free survival (DMFS), disease-free survival (DFS), and OS.
Multivariate analyses with the Cox proportional hazards model were used to test for independent prognostic factors by backward elimination of the insignificant explanatory variables. The Cox proportional hazards model was also used to calculate the hazard ratios (HRs) and $95 \%$ confidence intervals (CIs). Host factors (age and sex) were included as covariates in all tests. Two-tailed $P$ values $<0.05$ were considered statistically significant.

\section{Results}

\section{Patient characteristics}

The median patient age was 43 years (range, 13-78 years), and the cohort included 580 males and 169 females (male:female ratio of 3.4:1). Histologically, 744 (99.3\%) patients had World Health Organization (WHO) type II/III disease, and 5 (0.7\%) had WHO type I disease. Positron emission tomography (PET)/ CT was performed for $162(21.6 \%)$ patients. Using the 2009 7th UICC/AJCC staging system, the numbers of patients with stage I, II, III, IVa, and IVb disease were 78 (10.4\%), 179 (23.9\%), 282 (37.7\%), 160 (21.3\%), and $50(6.7 \%)$, respectively. The median follow-up duration was 81.4 months (range, 3.1-126.5 months). In total, $424(86.2 \%)$ of the 492 patients with stage III-IV disease received chemotherapy. The characteristics of the patients are summarized in Table 1.

\section{Dose-volume histogram analysis}

Table 2 summarizes the dose-volume histogram (DVH) statistics for the target volumes of interest. The maximum dose $\left(D_{\max }\right)$ and minimum dose $\left(D_{\min }\right)$ of radiation were defined as the maximum and minimum dose points in the DVH. The radiation dose prescribed to the PTV-P was $68 \mathrm{~Gy}$ for all patients. Because the proportion of the volume that received $<93 \%$ of the prescribed dose was set to $\leq 3 \%$, the actual median dose to the PTV-P was 73.34 Gy.

The dose limitations are $\leq 60$ Gy to the brainstem and $\leq 50$ Gy to the spinal cord. In some advanced diseases with extensive extension, we would relax the dose limitation to $D_{\max } \leq 63$ Gy. The median $D_{\max }$ for the brainstem was $54.16 \mathrm{~Gy}$ (range, 31.68-77.65 Gy), and the median proportion of the volume in the brainstem that received $>60$ Gy was $9.67 \%$ (range, $0.03 \%-26.97 \%$ ). The median $\mathrm{D}_{\max }$ for the spinal cord was 33.96 Gy (range, 8.29-60.54 Gy), and the median proportion of the volume in the spinal cord that received $>50$ Gy was $0.97 \%$ (range, $0.01 \%-3.42 \%$ ). However, 3 of the 749 patients received irradiation with a $D_{\max }>63 \mathrm{~Gy}$, with no serious late toxicity for the brainstem. The median doses to the left and right parotid glands were 28.20 Gy (range, 11.90 49.70 Gy) and 29.98 Gy (range, 21.30-42.02 Gy). The median proportions of the volumes in the left and right 
Table 1 Clinicopathologic features of the 749 patients with nasopharyngeal carcinoma (NPC)

\begin{tabular}{lc}
\hline Feature & No. of patients [cases (\%)] \\
\hline Sex & \\
Male & $580(77.4)$ \\
Female & $169(22.6)$ \\
WHO pathology & \\
Type I & $5(0.7)$ \\
Type II/II & $744(99.3)$ \\
Clinical stage & \\
I-II & $257(34.3)$ \\
III-IV & $492(65.7)$ \\
T stage & \\
T1 & $177(23.6)$ \\
T2 & $140(18.7)$ \\
T3 & $264(35.2)$ \\
T4 & $168(22.4)$ \\
N stage & \\
N0 & $184(24.6)$ \\
N1 & $409(54.6)$ \\
N2 & $106(14.2)$ \\
N3 & $50(6.7)$ \\
Chemotherapy & \\
No chemotherapy & $214(28.6)$ \\
Concurrent & $243(32.5)$ \\
Induction + concurrent & $246(32.8)$ \\
Concurrent + adjuvant & $46(6.1)$ \\
\hline WHOWold Heath Organiaton
\end{tabular}

WHO World Health Organization

a According to the 7th edition of the Union for International Cancer Control/ American Joint Committee on Cancer (UICC/AJCC) staging system

parotid glands that received a dose $>26$ Gy were $49.34 \%$ (range, 9.61\%-98.25\%) and 51.23\% (20.59\%-96.61\%).

\section{Patterns of treatment failure and survival}

Among the 749 patients, 56 (7.5\%) relapsed [34 (4.5\%) had local relapse only, 15 (2.0\%) had nodal relapse only, 7 (1.0\%) had both local and nodal relapse], and 129 (17.2\%) had distant metastasis. One hundred forty-nine (19.9\%) patients died. The median time to relapse was 25.2 (range, 7.0-93.8) months, and the median time to distant metastases was 18.9 (range, 2.6-92.1) months.
The 5-year local failure, regional failure, locoregional failure, and distant failure rates for the whole cohort were 5.4, 3.0, 7.4, and $17.4 \%$, respectively (Fig. 1 ). The 5 -year survival rates for the whole cohort were as follows: LRFS, 94.6\%; NRFS, 97.0\%; DMFS, 82.6\%; DFS, 75.1\%; and OS, $82.0 \%$. Using the 7 th edition of the UICC/AJCC staging system, the 5-year LRFS rates for patients with stage T1-T4 NPC were 98.4, 96.2, 93.0, and 90.5\%, respectively (Fig. 2a); the 5-year DMFS rates for patients with stage N0-N3b NPC were 93.3, 84.2, 72.4, 61.9, and 50.7\%, respectively (Fig. 2b); and the 5 -year OS rates for patients with stage I-IVb NPC were 97.4, 93.8, 81.8, 69.7, and $54.0 \%$, respectively (Fig. 2c).

\section{Prognostic factors}

Table 3 lists the factors with independent significance for different endpoints. The TNM stage was consistently the most significant factor for prognosis prediction: $\mathrm{T}$ stage for local failure prediction $(P<0.001)$, and both $\mathrm{T}$ and $\mathrm{N}$ stages for distant failure, disease failure, and death prediction (all $P<0.001$ ). None of the factors had a significant influence on nodal failure. An age $>50$ years was associated with significantly higher rates of distant failure $(\mathrm{HR}=1.530,95 \% \mathrm{CI} 1.037-2.256, P=0.032)$, disease failure $(\mathrm{HR}=1.896,95 \% \mathrm{CI} 1.403-2.561, P<0.001)$, and death $(\mathrm{HR}=2.482,95 \%$ CI $1.770-3.482, P<0.001)$.

The involvement of the nasal cavity, oropharynx, parapharynx, skull base, paranasal sinus, cranial nerve palsy, intracranial extension, infratemporal fossa, intracranial extension, orbit, and hypopharynx (all were $\mathrm{T}$ stage-related parameters) occurred in 280 (37.4\%), 85 (11.3\%), 540 (72.1\%), 429 (57.3\%), 36 (4.8\%), 35 (4.7\%), and $3(0.4 \%)$ patients, respectively. To explore the prognostic values of the specific anatomic factors included in the TNM staging system, we analyzed all T stagerelated parameters, all $\mathrm{N}$ stage-related parameters (i.e., retropharyngeal lymph node involvement as well as the laterality, longest diameter, and Ho's location of the involved cervical lymph nodes), age, histological type, and chemotherapy modality using univariate and multivariate analyses. With the exception of hypopharyngeal involvement, the univariate analysis revealed that age, histological type, and all $\mathrm{T}$ stage- and $\mathrm{N}$ stage-related parameters were significantly associated with disease

Table 2 Dose-volume histogram data for planning target volumes in the 749 patients with NPC

\begin{tabular}{lllll}
\hline Variate & Volume $(\mathbf{m L})$ & $\mathbf{D}_{\max }(\mathrm{Gy})$ & $\mathbf{D}_{\min }(\mathrm{Gy})$ & \multicolumn{1}{c}{ Mean dose (Gy) } \\
\hline PTV-P & $51.89(2.60-287.48)$ & $79.91(70.47-93.95)$ & $59.79(50.58-71.16)$ & $73.11(68.16-78.48)$ \\
PTV1 & $97.35(13.78-494.77)$ & $79.67(72.81-86.08)$ & $58.86(49.13-67.32)$ & $67.94(56.66-74.48)$ \\
PTV2 & $368.97(106.36-685.58)$ & $78.99(70.04-86.74)$ & $45.28(43.12-61.47)$ & $60.55(53.64-72.13)$ \\
\hline
\end{tabular}

PTV-P planning target volume of the primary tumor, PTV1 PTV of the high-risk clinical target volume, PTV2 PTV of the low-risk clinical target volume, $D_{\max }$ maximum dose, $D_{\min }$ minimum dose. All data are presented as median with range in parentheses 


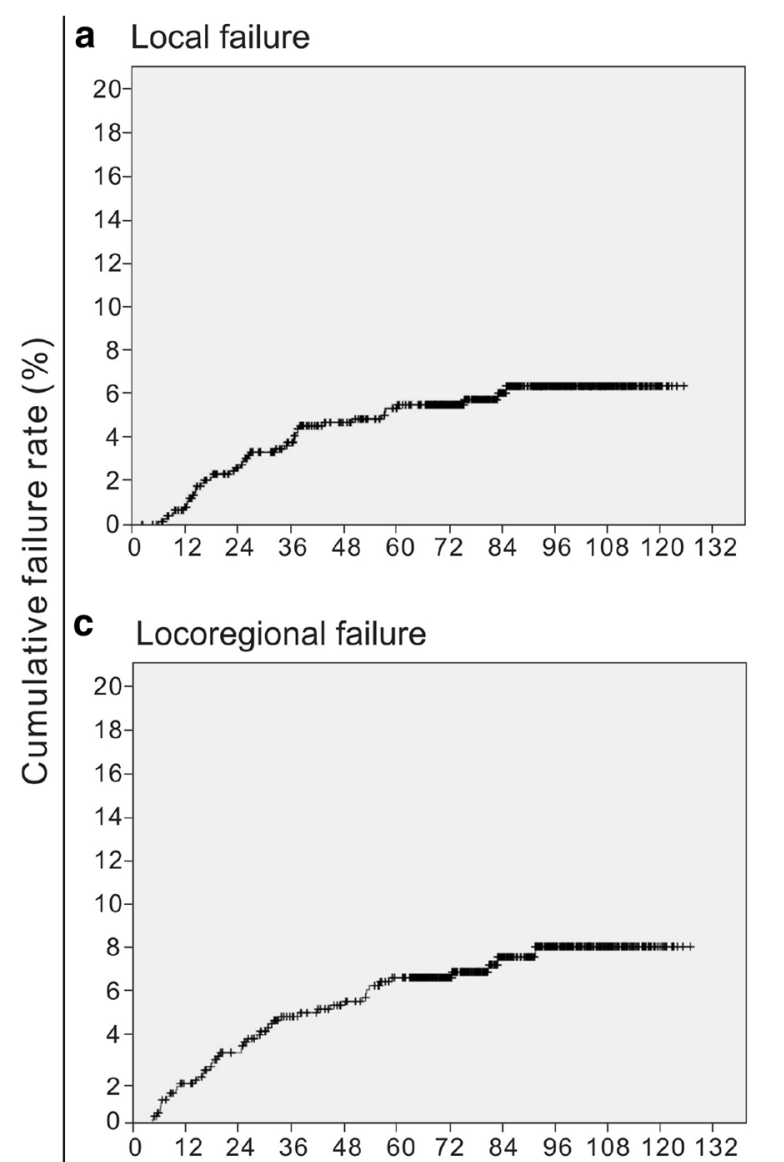

b Regional failure

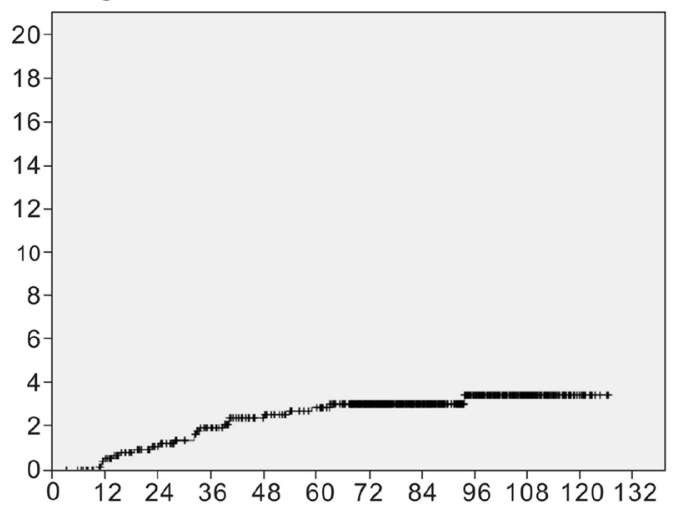

d Distant failure

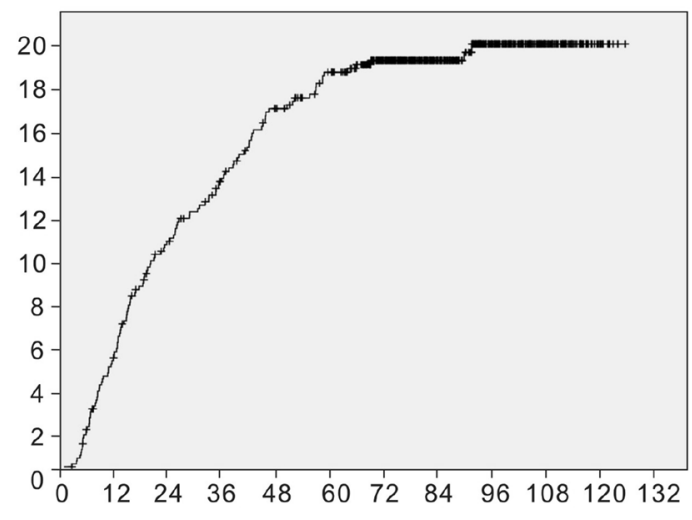

Time after treatment initiation (months)

Fig. 1 Cumulative local failure $(\mathbf{a})$, regional failure $(\mathbf{b})$, locoregional failure $(\mathbf{c})$, and distant failure $(\mathbf{d})$ rates of 749 patients with nasopharyngeal carcinoma (NPC) who were treated with intensity-modulated radiation therapy (IMRT)

failure (all $P<0.05$ ). All factors that were significant in univariate analysis were included in the Cox proportion hazards model with the backward elimination of nonsignificant explanatory variables. Only orbit involvement was found to be significant prognostic factor for local failure in the multivariate analysis $(P=0.011)$. Parapharyngeal involvement, retropharyngeal lymph node involvement, and the laterality, longest diameter, and Ho's location of the involved cervical lymph nodes were significant prognostic factors for both distant failure and disease failure (all $P<0.05$ ). Intracranial extension had significant prognostic value for distant failure $(P=0.040)$ (Table 4$)$.

\section{Discussion}

The present study, with 5-year LRFS and DMFS rates of 94.6\% and $82.6 \%$, confirmed that distant metastasis has surpassed local relapse to be the major cause of failure in NPC in the IMRT era, whereas our previous data from the conventional radiotherapy era demonstrated that both local relapse and distant metastasis were the major causes of failure in NPC, with 5-year LRFS and DMFS rates of $85 \%$ and $81 \%$ [6]. Although $\mathrm{T}$ stage was still a significant predictor for local failure, with the more extensive primary extension associated with poorer local control, the 5-year LRFS rates for patients with NPC at adjacent $\mathrm{T}$ stages were not significantly different. $\mathrm{T}$ stage is becoming less powerful in segregating patients into various risk groups. Improvements in local control have reduced the significance of known prognostic factors, even the $\mathrm{T}$ stage, for local control prediction [9]. It has been recommended that three rather than four $\mathrm{T}$ stages should be applied to classify patients in the modern era $[19,20]$. In the future, treatment modality may be the only significant predictor for local control in NPC patients; and the most important challenge in the management of NPC should be the exploration of more effective systemic agents to control distant metastasis. 


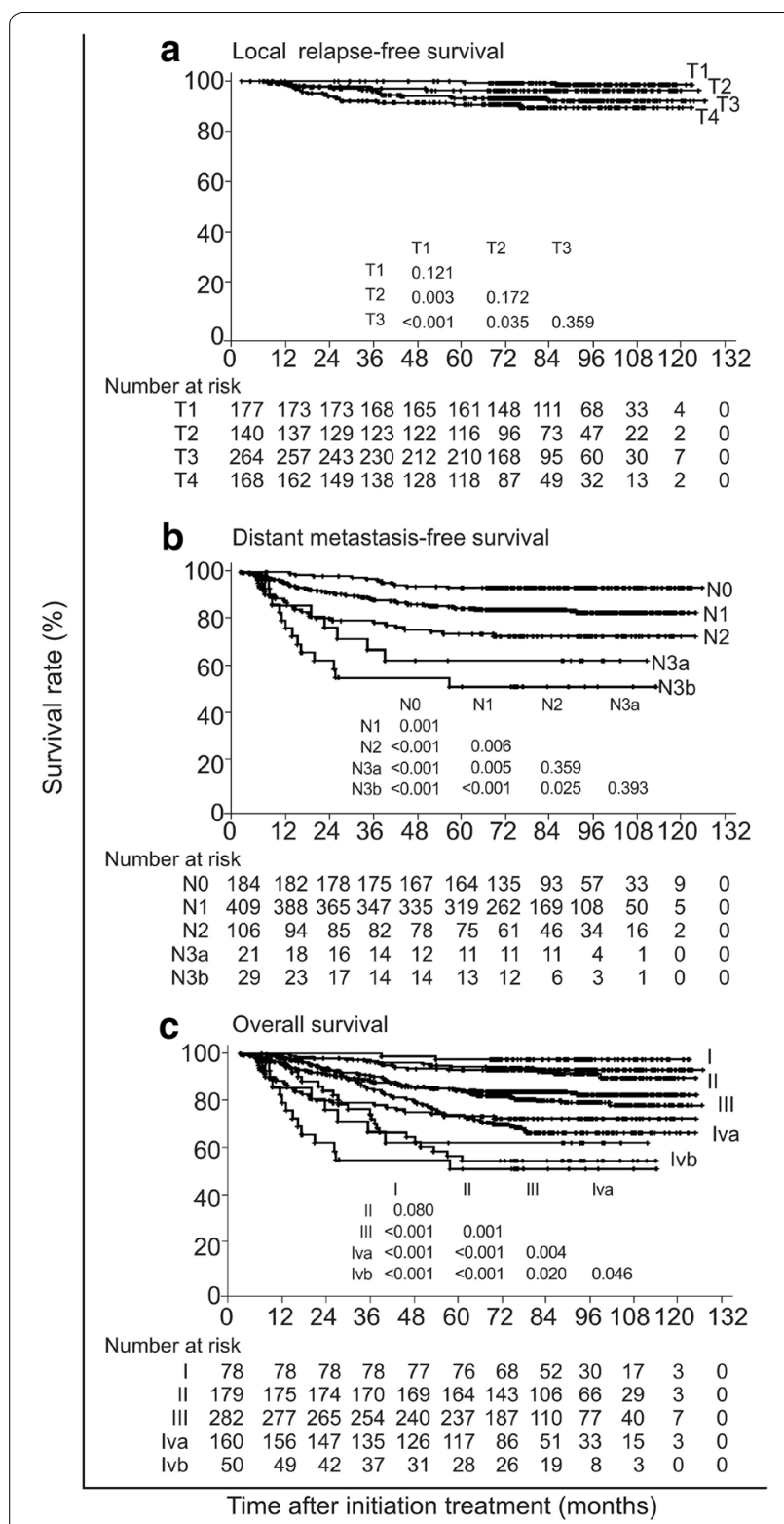

Fig. 2 Survival curves of 749 patients with NPC who were treated with IMRT. The current 7th edition of the Union for International Cancer Control/American Joint Committee on Cancer (UICC/AJCC) staging system was used for staging. a The 5-year local relapse-free survival (LRFS) rates for patients with stage T1-T4 NPC were 98.4, 96.2, 93.0, and 90.5\%, respectively. b The 5-year distant metastasis-free survival (DMFS) rates for patients with stage NO-N3b NPC were 93.3, $84.2,72.4,61.9$, and $50.7 \%$, respectively. c the 5 -year overall survival (OS) rates for patients with stage I-IVb NPC were 97.4, 93.8, 81.8, 69.7, and $54.0 \%$, respectively

Based on CT and conventional radiotherapy, several studies have systematically investigated prognostic factors for local control in patients with NPC [21-23]. Two studies have demonstrated that skull base extension or cranial nerve palsy could affect local failure-free survival
[21, 22], whereas one has confirmed that parapharyngeal tumor extension was an independent prognostic factor for local failure-free survival [23]. Based on MRI and IMRT, no systematic data referring to prognostic factors in patients with NPC were available. Zong et al. [24] focused on skull base extension and found that it did not influence local failure in the IMRT era. Based on the same database presented here, our previous studies reported that skull base extension [25] and parapharyngeal tumor extension [26] were not independent prognostic factors for local control in patients with NPC treated with IMRT. In the current study, we systematically re-evaluated all of the prognostic factors included in the TNM staging system and found that their prognostic values no longer remained significant and that orbit involvement was the only independent prognostic factor for local control.

Improvements in local control can explain these results. First, due to its improved dose conformability and escalation, IMRT eliminates the areas at the edges of the shielding blocks and field borders, such as the sphenoid sinus behind the pituitary shield, parapharyngeal region, and posterior skull base, which would be under-dosed when two-dimensional conventional radiotherapy is applied [27]. Thus, IMRT leads to improved local control in these areas. Second, MRI improves visualization and is superior to CT for identifying parapharyngeal tumor extension, skull base extension, and intracranial involvement [13]. Therefore, a more precise dose of radiation can be delivered to the gross target volume of primary tumor, which ultimately improves local control for these areas. However, orbit extension, which usually occurs as tumor extension from the pterygopalatine fossa via the inferior orbital fissure or directly from the cavernous sinus, is a marker of the most severe extension of NPC [15]. Even with the most sophisticated treatment techniques and intensive use of chemotherapy, some patients with advanced T4 disease that approaches important organs at risk cannot tolerate a radiation dose of $70 \mathrm{~Gy}$, and the local control rate for this subgroup remains low, even after IMRT [28]. Some patients who had tiny intracranial extensions (for example, through the oval foramen or foramen lacerum to cavernous sinus) or cranial nerve palsy and did not have disease approaching important organs at risk were still able to tolerate a radiation dose of $70 \mathrm{~Gy}$ and achieve excellent local control. This finding might explain why orbit involvement alone and not intracranial extension or cranial nerve palsy was the only independent prognostic factor for local control.

In patients diagnosed using $\mathrm{CT}$ and treated with conventional radiotherapy, parapharyngeal tumor extension, skull base extension, cranial nerve palsy, retropharyngeal lymph node involvement, and all cervical lymph noderelated parameters (i.e., laterality, size, Ho's location, and 


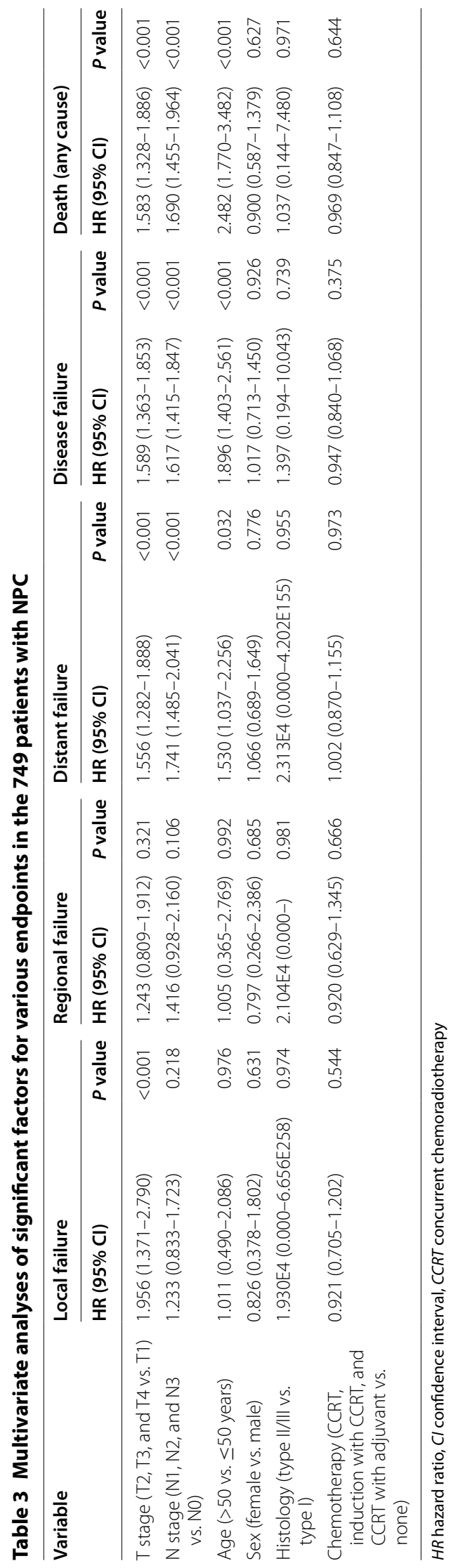


Table 4 Multivariate prognostic analyses of anatomic factors in the 749 patients with NPC

\begin{tabular}{|c|c|c|c|c|c|c|}
\hline \multirow[t]{2}{*}{ Variable } & \multicolumn{2}{|l|}{ Disease failure } & \multicolumn{2}{|l|}{ Local failure } & \multicolumn{2}{|l|}{ Distant failure } \\
\hline & $\mathrm{HR}(95 \% \mathrm{Cl})$ & $P$ value & $\mathrm{HR}(95 \% \mathrm{Cl})$ & $P$ value & $\mathrm{HR}(95 \% \mathrm{Cl})$ & $P$ value \\
\hline Age (>50 vs. $\leq 50$ years) & $1.857(1.365-2.516)$ & $<0.001$ & $0.996(0.476-2.080)$ & 0.997 & $1.433(0.967-2.120)$ & 0.074 \\
\hline Histological type (WHO II/III vs. WHO I) & $1.371(0.829-2.264)$ & 0.221 & $0.843(0.327-2.125)$ & 0.710 & $3.614(1.804-7.219)$ & $<0.001$ \\
\hline $\begin{array}{l}\text { Chemotherapy (CCRT, induction with CCRT, and CCRT } \\
\text { with adjuvant vs. none) }\end{array}$ & $1.207(0.794-1.843)$ & 0.383 & $0.624(0.273-1.410)$ & 0.269 & $1.459(0.834-2.569)$ & 0.190 \\
\hline Nasal cavity involvement (yes vs. no) & $0.946(0.683-1.311)$ & 0.741 & $0.867(0.429-1.752)$ & 0.690 & $0.818(0.547-1.225)$ & 0.330 \\
\hline Oropharyngeal involvement (yes vs. no) & $1.208(0.812-1.798)$ & & $0.431(0.130-1.432)$ & 0.169 & $1.491(0.941-2.362)$ & 0.089 \\
\hline Parapharyngeal involvement (yes vs. no) & $1.843(1.144-2.969)$ & 0.011 & $2.212(0.690-7.141)$ & 0.172 & $2.040(1.115-3.764)$ & 0.017 \\
\hline Skull base involvement (yes vs. no) & $1.273(0.851-1.906)$ & 0.240 & $2.113(0.790-5.651)$ & 0.136 & $1.248(0.759-2.052)$ & 0.383 \\
\hline Paranasal sinus involvement (yes vs. no) & $1.078(0.704-1.650)$ & 0.729 & $1.246(0.526-2.949)$ & 0.617 & $0.937(0.543-1.616)$ & 0.815 \\
\hline Cranial nerve involvement (yes vs. no) & $1.394(0.867-2.178)$ & 0.139 & $2.012(0.833-4.881)$ & 0.120 & $1.247(0.703-2.218)$ & 0.399 \\
\hline Intracranial involvement (yes vs. no) & $1.303(0.871-1.950)$ & 0.198 & $1.119(0.468-2.674)$ & 0.800 & $1.538(1.056-2.490)$ & 0.040 \\
\hline Infratemporal fossa involvement (yes vs. no) & $1.035(0.564-1.875)$ & 0.893 & $0.616(0.175-2.137)$ & 0.458 & $0.737(0.320-1.675)$ & 0.467 \\
\hline Orbit involvement (yes vs. no) & $1.641(0.914-2.937)$ & 0.109 & $4.067(1.369-12.103)$ & 0.011 & $1.537(0.733-3.231)$ & 0.251 \\
\hline Retropharyngeal lymph node involvement (yes vs. no) & $1.583(1.102-2.273)$ & 0.011 & $1.519(0.679-3.406)$ & 0.309 & $1.565(1.078-2.499)$ & 0.030 \\
\hline \multicolumn{7}{|l|}{ Cervical lymph node involvement } \\
\hline Longest diameter ( $>6 \mathrm{~cm}$ vs. $\leq 6 \mathrm{~cm}$ ) & $2.829(1.603-4.991)$ & $<0.001$ & $0.815(0.107-6.219)$ & 0.840 & $3.050(1.625-5.727)$ & 0.001 \\
\hline Laterality (bilateral vs. unilateral) & $1.475(1.031-2.107)$ & 0.032 & $0.398(0.672-6.513)$ & 0.423 & $1.625(1.078-2.453)$ & 0.019 \\
\hline Ho's location (Ho's vs. above Ho's) & $2.396(1.435-3.996)$ & 0.001 & $0.567(0.073-4.272)$ & 0.583 & $2.859(1.617-5.061)$ & $<0.001$ \\
\hline
\end{tabular}

$H R$ hazard ratio, $\mathrm{Cl}$ confidence interval, CCRT current chemoradiotherapy

fixity) were proved to be significant prognostic factors for distant control [22, 29]. In the present study, patients were diagnosed using MRI and treated with IMRT, and parapharyngeal tumor extension and all $\mathrm{N}$ stage-related parameters (i.e., retropharyngeal lymph node involvement as well as the laterality, size, and Ho's location of involved cervical lymph nodes) remained prognostic significance for DMFS. A number of studies have confirmed that IMRT has improved local control but not distant control in NPC $[8,10,28]$. Thus, most of the parameters that influence distant control have not been altered by the introduction of IMRT.

Compared with previous studies, one major different finding in the present study is that intracranial extension rather than cranial nerve palsy was a significant prognostic factor for distant control. Intracranial extension, which was defined as the involvement of the cavernous sinus, brain tissue, cistern, or dural meninges and could not be easily detected by $\mathrm{CT}$, could be demonstrated more precisely by MRI because of its better tissue contrast and multiplanar capacity [13]. It has been reported that most patients with clinical cranial nerve palsy display one or more sites of intracranial involvement on MRI [30]. Therefore, the previously demonstrated adverse prognostic significance of cranial nerve palsy may be accurately reflected by intracranial extension on MRI.

Another difference was the lack of significant prognostic value for skull base extension in terms of DMFS in the present study. It has been reported that $51.2 \%$ of patients with skull base extension shown on MRI but not on CT images had minimal bone disease [13]. These patients would have a more favorable prognosis [31,32]. Although the diagnosis of skull base extension based entirely on MRI leads to an upstaging according to the UICC/AJCC staging system, skull base extension has not been confirmed as a significant prognostic factor for DMFS. However, the prognostic value of MRI-detected skull base extension varies in different studies; although MRI-detected skull base extension was not an independent prognostic factor, the grading of skull base extension according to the site of extension has previously been reported to be an independent prognostic factor for both OS and DMFS [33].

The prognosis of a patient with cancer depends on the biological aggressiveness of the tumor, host factors, and therapeutic interventions [9]. However, we explored the prognosis only according to anatomic factors in this study. An increasing number of biological, genetic, and molecular factors, such as plasma/serum Epstein-Barr virus (EBV) DNA [34] and specific microRNAs (miRNAs) [35], as well as the target volume [36, 37] and other non-anatomic factors, have been identified and studied [38]. Such factors may have profound influences on the prognosis for individual patients. However, the extent of local extension, regional lymphatic spread, and distant metastasis as reflected by TNM staging still remain the most important prognostic factors in NPC [9]. 
Because the definition of parapharyngeal tumor extension has differed substantially across different time points $[26,39]$, it is difficult to compare the prognostic value of parapharyngeal tumor extension. This was a retrospective study that aimed to evaluate the prognostic value of staging parameters amid the changes in diagnostic and therapeutic methods. Moreover, due to financial factors and some patients' lack of health insurance, PET/CT was only performed on 130 (25.4\%) of the 749 patients in our study. Additional effects of sensitive and specific imaging technologies such as PET on stage migration are expected. With its well-recognized superiority in the detection of nodal metastases and distant metastases [40, 41], there seems to be little doubt that the addition of PET (with or without CT) could contribute to improve staging accuracy and prognostication in NPC.

\section{Conclusions}

The key failure pattern for NPC in the IMRT era was distant metastasis. With the changes in diagnostic and therapeutic technologies, the significant prognostic parameters for local control have also altered substantially. Orbit involvement was the only independent prognostic factor for local control, and intracranial extension, parapharyngeal tumor extension, and all $\mathrm{N}$ stage-related parameters were independent prognostic factors for DMFS.

\section{Authors' contributions \\ Conception and design of the study: YPM, LLT and JM Acquisition of data: YM and LZL Analysis and interpretation of the data:YPM, LLT, JM and AHL Contrib- uted reagents/materials/analysis tools: All authors. Writing and revision of the manuscript: All authors. All authors read and approved the final manuscript.}

\section{Author details \\ ${ }^{1}$ State Key Laboratory of Oncology in South China, Collaborative Innovation Center for Cancer Medicine, Sun Yat-sen University Cancer Center, Guang- zhou 510060, Guangdong, P. R. China. ${ }^{2}$ Department of Radiation Oncology, Sun Yat-sen University Cancer Center, Guangzhou 510060, Guangdong, P. R. China. ${ }^{3}$ Imaging Diagnosis and Interventional Center, Sun Yat-sen University Cancer Center, Guangzhou 510060, Guangdong, P. R. China. ${ }^{4}$ Department of Medical Statistics and Epidemiology, School of Public Health, Sun Yat-sen University, Guangzhou 510080, Guangdong, P. R. China.}

\section{Acknowledgements \\ This work was supported by grants from the Key Laboratory Construction Pro- ject of Guangzhou City, China (121800085), the Health \& Medical Collaborative Innovation Project of Guangzhou City, China (201400000001), the National Science \& Technology Pillar Program during the Twelfth Five-year Plan Period (2014BAl09B10), the National Natural Science Foundation of China (81201746), and the Planned Science and Technology Project of Guangdong Province, China (2013B020400004) \\ We received writing assistance from a professional English editing service that specializes in scientific papers at Elixigen Corporation.}

\section{Competing interests}

The authors declare that they have no competing interests.

Received: 18 August 2015 Accepted: 12 July 2016

Published online: 28 December 2016
References

1. Gibb AG, Van Hasselt CA. Nasopharyngeal carcinoma, vol xvii. Hong Kong: Chinese University Press; Greenwich Medical Media; 1999. p. 337.

2. Moss WT. Therapeutic radiology: rationale, technique, results, vol xviii. St. Louis: C.V. Mosby Co; 1965. p. 514.

3. Lee AW, Poon YF, Foo W, Law SC, Cheung FK, Chan DK, et al. Retrospective analysis of 5037 patients with nasopharyngeal carcinoma treated during 1976-1985: overall survival and patterns of failure. Int J Radiat Oncol Biol Phys. 1992;23(2):261-70.

4. Geara FB, Sanguineti G, Tucker SL, Garden AS, Ang KK, Morrison WH, et al. Carcinoma of the nasopharynx treated by radiotherapy alone: determinants of distant metastasis and survival. Radiother Oncol. 1997:43(1):53-61.

5. Lee AW, Sze WM, Au JS, Leung SF, Leung TW, Chua DT, et al. Treatment results for nasopharyngeal carcinoma in the modern era: the Hong Kong experience. Int J Radiat Oncol Biol Phys. 2005;61 (4):1107-16. doi:10.1016/j.jijobp.2004.07.702.

6. Liu MZ, Tang LL, Zong JF, Huang Y, Sun Y, Mao YP, et al. Evaluation of sixth edition of AJCC staging system for nasopharyngeal carcinoma and proposed improvement. Int J Radiat Oncol Biol Phys. 2008;70(4):1115-23. doi:10.1016/j.ijrobp.2007.07.2353.

7. Lee N, Harris J, Garden AS, Straube W, Glisson B, Xia P, et al. Intensity-modulated radiation therapy with or without chemotherapy for nasopharyngeal carcinoma: radiation therapy oncology group phase II trial 0225. J Clin Oncol. 2009;27(22):3684-90. doi:10.1200/jco.2008.19.9109.

8. Ng WT, Lee MC, Hung WM, Choi CW, Lee KC, Chan OS, et al. Clinical outcomes and patterns of failure after intensity-modulated radiotherapy for nasopharyngeal carcinoma. Int J Radiat Oncol Biol Phys. 2011;79(2):4208. doi:10.1016/j.jijobp.2009.11.024.

9. Lu JJ, Lee AWM, Cooper JS, SpringerLink. Nasopharyngeal cancer multidisciplinary management. Medical radiology, diagnostic imaging and radiation oncology. Berlin: Springer; 2010.

10. Lai SZ, Li WF, Chen L, Luo W, Chen YY, Liu LZ, et al. How does intensitymodulated radiotherapy versus conventional two-dimensional radiotherapy influence the treatment results in nasopharyngeal carcinoma patients? Int J Radiat Oncol Biol Phys. 2011;80(3):661-8. doi:10.1016/j. ijrobp.2010.03.024.

11. Greene FL, American Joint Committee on C, American Cancer S. AJCC cancer staging manual. Cancer staging manual, vol xiv. New York: Springer; 2002. p. 421

12. King AD, Lam WW, Leung SF, Chan YL, Teo P, Metreweli C. MRI of local disease in nasopharyngeal carcinoma: tumour extent vs tumour stage. $\mathrm{Br}$ J Radiol. 1999;72(860):734-41. doi:10.1259/bjr.72.860.10624338.

13. Liao XB, Mao YP, Liu LZ, Tang LL, Sun Y, Wang Y, et al. How does magnetic resonance imaging influence staging according to AJCC staging system for nasopharyngeal carcinoma compared with computed tomography? Int J Radiat Oncol Biol Phys. 2008;72(5):1368-77. doi:10.1016/j. ijrobp.2008.03.017.

14. Heng DM, Wee J, Fong KW, Lian LG, Sethi VK, Chua ET, et al. Prognostic factors in 677 patients in Singapore with nondisseminated nasopharyngeal carcinoma. Cancer. 1999;86(10):1912-20.

15. King AD, Bhatia KS. Magnetic resonance imaging staging of nasopharyngeal carcinoma in the head and neck. World J Radiol. 2010;2(5):159-65. doi:10.4329/wjr.v2.15.159.

16. Hsu WM, Wang AG. Nasopharyngeal carcinoma with orbital invasion. Eye (London, England). 2004;18(8):833-8. doi:10.1038/sj.eye.6701358.

17. Teresi LM, Lufkin RB, Vinuela F, Dietrich RB, Wilson GH, Bentson JR, et al. MR imaging of the nasopharynx and floor of the middle cranial fossa. Part II. Malignant tumors. Radiology. 1987;164(3):817-21. doi:10.1148/ radiology.164.3.3615884.

18. van den Brekel MW, Stel HV, Castelijns JA, Nauta JJ, van der Waal I, Valk J, et al. Cervical lymph node metastasis: assessment of radiologic criteria. Radiology. 1990;177(2):379-84. doi:10.1148/radiology.177.2.2217772.

19. Lee AW, Ng WT, Chan LK, Chan OS, Hung WM, Chan CC, et al. The strength/weakness of the AJCC/UICC staging system (7th edition) for nasopharyngeal cancer and suggestions for future improvement. Oral Oncol. 2012;48(10):1007-13. doi:10.1016/j.oraloncology.2012.03.022.

20. Chen L, Mao YP, Xie FY, Liu LZ, Sun Y, Tian L, et al. The seventh edition of the UICC/AJCC staging system for nasopharyngeal carcinoma is prognostically useful for patients treated with intensity-modulated radiotherapy 
from an endemic area in China. Radiother Oncol. 2012;104(3):331-7. doi:10.1016/j.radonc.2011.10.009.

21. Sham JS, Choy D. Prognostic value of paranasopharyngeal extension of nasopharyngeal carcinoma on local control and short-term survival. Head Neck. 1991;13(4):298-310.

22. Teo P, Yu P, Lee WY, Leung SF, Kwan WH, Yu KH, et al. Significant prognosticators after primary radiotherapy in 903 nondisseminated nasopharyngeal carcinoma evaluated by computer tomography. Int J Radiat Oncol Biol Phys. 1996:36(2):291-304.

23. Chua DT, Sham JS, Kwong DL, Choy DT, Au GK, Wu PM. Prognostic value of paranasopharyngeal extension of nasopharyngeal carcinoma. A significant factor in local control and distant metastasis. Cancer. 1996;78(2):20210. doi:10.1002/(sici) 1097-0142(19960715)78:2<202:aid-cncr3>3.0.co;2-n.

24. Zong J, Lin S, Lin J, Tang L, Chen B, Zhang M, et al. Impact of intensitymodulated radiotherapy on nasopharyngeal carcinoma: validation of the 7th edition AJCC staging system. Oral Oncol. 2015;51(3):254-9. doi:10.1016/j.oraloncology.2014.10.012.

25. Cheng YK, Liu LZ, Jiang N, Yue D, Tang LL, Zhang F, et al. MRI-detected skull-base invasion: prognostic value and therapeutic implication in intensity-modulated radiotherapy treatment for nasopharyngeal carcinoma. Strahlentherapie und Onkologie: Organ der Deutschen Rontgengesellschaft [et al]. 2014;190(10):905-11. doi:10.1007/ s00066-014-0656-7.

26. Tang LL, Sun Y, Mao YP, Chen Y, Li WF, Chen L, et al. Prognostic value of parapharyngeal extension in nasopharyngeal carcinoma treated with intensity modulated radiotherapy. Radiother Oncol. 2014;110(3):404-8. doi:10.1016/j.radonc.2013.10.007.

27. Kam MK, Chau RM, Suen J, Choi PH, Teo PM. Intensity-modulated radiotherapy in nasopharyngeal carcinoma: dosimetric advantage over conventional plans and feasibility of dose escalation. Int J Radiat Oncol Biol Phys. 2003;56(1):145-57.

28. Cheng SH, Yen KL, Jian JJ, Tsai SY, Chu NM, Leu SY, et al. Examining prognostic factors and patterns of failure in nasopharyngeal carcinoma following concomitant radiotherapy and chemotherapy: impact on future clinical trials. Int J Radiat Oncol Biol Phys. 2001;50(3):717-26.

29. Lee AW, Foo W, Poon YF, Law CK, Chan DK, SK O, et al. Staging of nasopharyngeal carcinoma: evaluation of N-staging by Ho and UICC/AJCC systems. Union Internationale Contre le Cancer. American Joint Committee for Cancer. Clin Oncol (R Coll Radiol). 1996;8(3):146-54.

30. Liu L, Liang S, Li L, Mao Y, Tang L, Tian L, et al. Prognostic impact of magnetic resonance imaging-detected cranial nerve involvement in nasopharyngeal carcinoma. Cancer. 2009;115(9):1995-2003. doi:10.1002/ cncr.24201.
31. Lu JC, Wei BQ, Chen WZ, Qian PD, Zhang YQ, Wei Q, et al. Staging of nasopharyngeal carcinoma investigated by magnetic resonance imaging. Radiother Oncol. 2006;79(1):21-6. doi:10.1016/j.radonc.2006.03.015.

32. Altun M, Tenekeci N, Kaytan E, Meral R. Locally advanced nasopharyngeal carcinoma: computed tomography findings, clinical evaluation, and treatment outcome. Int J Radiat Oncol Biol Phys. 2000;47(2):401-4.

33. Chen L, Liu LZ, Mao YP, Tang LL, Sun Y, Chen Y, et al. Grading of MRIdetected skull-base invasion in nasopharyngeal carcinoma and its prognostic value. Head Neck. 2011;33(9):1309-14. doi:10.1002/hed.21606.

34. Tang LQ, Chen QY, Fan W, Liu H, Zhang L, Guo L, et al. Prospective study of tailoring whole-body dual-modality [18F]fluorodeoxyglucose positron emission tomography/computed tomography with plasma Epstein-Barr virus DNA for detecting distant metastasis in endemic nasopharyngeal carcinoma at initial staging. J Clin Oncol. 2013;31 (23):2861-9. doi:10.1200/ jco.2012.46.0816.

35. Liu N, Chen NY, Cui RX, Li WF, Li Y, Wei RR, et al. Prognostic value of a microRNA signature in nasopharyngeal carcinoma: a microRNA expression analysis. Lancet Oncol. 2012;13(6):633-41. doi:10.1016/ s1470-2045(12)70102-X.

36. Guo R, Sun Y, Yu XL, Yin WJ, Li WF, Chen YY, et al. Is primary tumor volume still a prognostic factor in intensity modulated radiation therapy for nasopharyngeal carcinoma? Radiother Oncol. 2012;104(3):294-9. doi:10.1016/j.radonc.2012.09.001.

37. Tian YM, Xiao WW, Bai L, Liu XW, Zhao C, Lu TX, et al. Impact of primary tumor volume and location on the prognosis of patients with locally recurrent nasopharyngeal carcinoma. Chin J Cancer. 2015;34(6):247-53. doi:10.1186/s40880-015-0019-5.

38. Chen YP, Zhao BC, Chen C, Shen LJ, Gao J, Mai ZY, et al. Pretreatment platelet count improves the prognostic performance of the TNM staging system and aids in planning therapeutic regimens for nasopharyngeal carcinoma: a single-institutional study of 2,626 patients. Chin J Cancer. 2015;34(3):137-46. doi:10.1186/s40880-015-0006-x.

39. Sham JS, Cheung YK, Choy D, Chan FL, Leong L. Nasopharyngeal carcinoma: CT evaluation of patterns of tumor spread. AJNR Am J Neuroradiol. 1991;12(2):265-70.

40. Ng SH, Chang JT, Chan SC, Ko SF, Wang HM, Liao CT, et al. Nodal metastases of nasopharyngeal carcinoma: patterns of disease on MRI and FDG PET. Eur J Nucl Med Mol Imaging. 2004;31 (8):1073-80. doi:10.1007/ s00259-004-1498-9.

41. Yen TC, Chang JT, Ng SH, Chang YC, Chan SC, Lin KJ, et al. The value of 18F-FDG PET in the detection of stage M0 carcinoma of the nasopharynx. J Nucl Med. 2005:46(3):405-10.

\section{Submit your next manuscript to BioMed Central and we will help you at every step:}

- We accept pre-submission inquiries

- Our selector tool helps you to find the most relevant journal

- We provide round the clock customer support

- Convenient online submission

- Thorough peer review

- Inclusion in PubMed and all major indexing services

- Maximum visibility for your research

Submit your manuscript at www.biomedcentral.com/submit
(O) BioMed Central 